\title{
Bushen Huoxue decoction improves cognitive decline in rats with cerebral hypoperfusion
}

\author{
SHUIFEN YE ${ }^{1}$, YONG GU $^{2}$, YIHUI XU ${ }^{1}$, WEN FAN $^{1}$, XIAOTING WANG ${ }^{3}$, \\ SHUTING CHEN $^{3}$, SIYI CAI ${ }^{3}$, SHIRONG LV ${ }^{3}$, YAO TONG ${ }^{2}$ and JING CAI ${ }^{1}$ \\ ${ }^{1}$ Academy of Integrative Medicine, Fujian University of Traditional Chinese Medicine, \\ Fuzhou, Fujian 350122; ${ }^{2}$ School of Chinese Medicine, The University of Hong Kong, \\ Hong Kong SAR; ${ }^{3}$ College of Integrative Medicine, Fujian University of \\ Traditional Chinese Medicine, Fuzhou, Fujian 350122, P.R. China
}

Received July 27, 2013; Accepted April 29, 2014

DOI: $10.3892 / \mathrm{mmr} .2014 .2355$

\begin{abstract}
Cerebral hypoperfusion is a common feature of vascular dementia and has recently been recognized to contribute to the progression of cognitive decline. The present study aimed to investigate the effects of Bushen Huoxue decoction (BHD), a two-herb Chinese Medicine, on cognitive impairment in a rat model of cerebral hypoperfusion induced by permanent occlusion of bilateral common carotid arteries (2VO). The results demonstrated that BHD significantly attenuated learning and spatial memory deficits in the Morris water maze test in a dose-dependent manner. Transmission electron microscopy observation revealed that the reduction of synapse density in hippocampal CA1 and cortex parietal isolated from rats with $2 \mathrm{VO}$ was partially restored by BHD treatment. In addition, the expression levels of a number of antioxidants, including superoxide dismutase, catalase (CAT), glutathine and glutathione peroxidase-1 (GPX-1) increased, whereas malondialdehyde decreased in the hippocampi of rats with 2VO following BHD treatment. Polymerase chain reaction and western blot analysis further confirmed that the GPx-1 and CAT expression increased in the BHD treatment group. In conclusion the results suggested that BHD has therapeutic potential to treat vascular dementia, which may be associated with synapse density and anti-oxidant activities in the hippocampus.
\end{abstract}

\section{Introduction}

Vascular cognitive impairment (VCI) is the phenotypic outcome of a cascade of events, involving vascular risk

Correspondence to: Professor Jing Cai, Academy of Integrative Medicine, Fujian University of Traditional Chinese Medicine, 1 Huatuo Road, Shangjie, Minhou, Fuzhou, Fujian 350122, P.R. China

E-mail: caij1@163.com

Key words: Bushen Huoxue decoction, vascular dementia, synapse density, antioxidant enzymes factors that lead to vascular disease, which causes vascular brain injury in networks important for cognitive functioning (1-4). VCI encompasses numerous conditions from vascular dementia (VaD) to mild cognitive impairment of vascular origin (5). Clinical studies have reported that cerebral hypoperfusion associated with cognitive dysfunctions has been identified in Alzheimer's disease (AD) and in patients with $\mathrm{VaD}(6,7)$.

The pathological mechanisms that underlie chronic cerebral hypoperfusion-induced dementia are yet to be identified. Reactive oxidative stress and inflammatory cytokines have important roles in brain impairment and dementia progression (8). In addition, experimental evidence has indicated that synapse loss occurs in $\mathrm{AD}$ and $\mathrm{VaD}$ patients and studies revealed a strong correlation between synapse reduction and the severity of dementia (9). Although an increasing number of mechanistic pathological studies focused on VaD and hypoperfusion, no effective pharmacological intervention has been approved by the Food and Drug Administration (Silver Spring, MD, USA) and used clinically.

Herbal medicines, including Traditional Chinese Medicines, have been used for a long time as an alternative method to treat cerebral vascular diseases and dementia-like symptoms (10). A Chinese Medicine named Bushen Huoxue decoction (BHD) was prepared, which has a good clinical efficacy in treating patients with $\mathrm{VaD}$. Two herbs are included in BHD, namely Epimedium (Epimedium Davidii Franch, Yin Yang Huo) and Salvia Miltrorrhiza (Salvia Miltiorrhiza Bunge, Dan Shen). BHD has the efficacy of nourishing kidney and activating blood (Bu Shen Huo Xue), which is simplified from an impactful formula based on the Traditional Chinese Medicine theory that has been previously confirmed to have good efficacy in treating cognitive impairment, ischemic cerebral stroke and $\mathrm{VaD}$ (11). Although the formula has been proved to be effective in the clinical treatment of $\mathrm{VaD}$, whether the simplified decoction BHD may improve cognitive deficits and the associated mechanism underlying its effects remain to be elucidated.

In the present study, occlusion of bilateral common carotid arteries (2VO) was used as a cerebral hypoperfusion model to study the effect of BHD on cognitive improvement in vivo. 


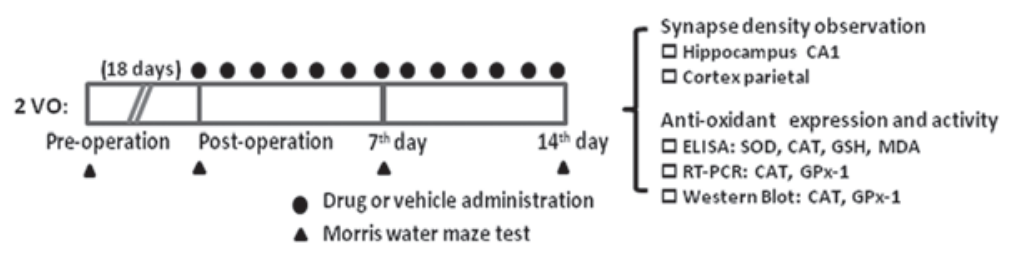

Figure 1. Outline of the experimental design illustrating the time scale of drug administration and Morris water maze test. The rats were subjected to permanent $2 \mathrm{VO}$ surgery. Following 18 days, the rats in each group were intragastrically administered twice daily with $2.75,5.5$ and $11 \mathrm{~g} / \mathrm{kg}$ Bushen Huoxue decoction, $5.85 \mathrm{mg} / \mathrm{kg}$ Ginkgo biloba or saline, respectively. The administration was performed for 14 consecutive days. The animals were subjected to behavioral testing prior to the surgery, post-surgery without treatment, and on days 7 and 14 following administration, respectively, followed by outcome parameters detection, including synapses number and anti-oxidants levels. 2VO, occlusion of bilateral common carotid arteries; GPx-1, glutathione peroxidase-1; CAT, catalase; SOD, superoxide dismutase; GSH, glutathione.

\section{Materials and methods}

Plant materials and herbs water extraction. BHD consists of Epimedium and Salvia miltiorrhiza. The herbs were purchased from Fujian Pharmaceutical Co. (Fuzhou, Fujian, China) and were identified as eligible and pure medicinal material. These herbs were decocted at boiling temperature and extracted with water. The decoction was filtrated, concentrated and stored in a sterile bottle at $4^{\circ} \mathrm{C}$ until use. An extract of Ginkgo biloba (GB) leaves was purchased from Shenyang Green Pharmaceutical Co. (Shenyang, Liaoning, China).

Animals and 2VO surgery. The current study was approved by the ethics committee of Fujian University of Traditional Chinese Medicine, Fuzhou, China. Wistar rats (Shanghai SLAC Laboratory Animal Co., Ltd., Shanghai, China), weighing $250 \pm 20 \mathrm{~g}$, were allowed to acclimatize for three days prior to experimentation and were housed in $25 \pm 1^{\circ} \mathrm{C}, 65 \pm 5 \%$ humidity and a 12 -h light/dark cycle. Food and water were freely available. All experiments were conducted in accordance with the Guidance Suggestions for the Care and Use of Laboratory Animals, formulated by the Ministry of Science and Technology of China. Efforts were made to minimize the suffering and number of animals used.

Cerebral hypoperfusion in rats was performed according to the previously described procedures (12). Each rat was anesthetized with ketamine hydrochloride $(0.3 \mathrm{ml} / 100 \mathrm{~g}$, i.p.). Both of the common carotid arteries were carefully separated from the cervical sympathetic and vagal nerves through a ventral cervical incision. The arteries were then ligated with 5.0 silk sutures. The sham-operated animals were treated similarly to the $2 \mathrm{VO}$ rats except for the carotid artery ligations.

Drug administration. The adult normal dosage of BHD was defined as a moderate dose $(\mathrm{M}), 1 / 2$ of the moderate dose as the low dose (L) and two times the moderate dose as the high dose $(\mathrm{H})$. On the day 18 following surgery, the $2 \mathrm{VO}$ rats were randomly divided into five groups with $n=12$ /group. The rats in each group were intragastrically administrated twice daily with saline (vehicle control, 2VO), $2.75 \mathrm{~g} / \mathrm{kg}$ (BHD-L), $5.5 \mathrm{~g} / \mathrm{kg}$ (BHD-M), $11 \mathrm{~g} / \mathrm{kg}$ (BHD-H) BHD and $5.85 \mathrm{mg} / \mathrm{kg}$ GB, respectively. GB, a well-known antioxidant and a good anti-dementia drug, was used as a positive control $(13,14)$. The sham-operated and vehicle groups were administered an equal volume of physiological saline solution. The administration was performed for
14 consecutive days. The animals were subjected to behavioral tests prior to the surgery, post-surgery and on days 7 and 14 following administration, respectively, to observe the learning and memory ability. The time scale of the drug treatment regimen is illustrated in Fig. 1.

Morris water maze tests (MWM). Prior to the surgery, post-surgery and on days 7 and 14 following treatment, respectively, the rats were subjected to spatial learning and memory tests as examined using the MWM as previously described (15). The MWM consisted of a black circular pool of water and a hidden platform submerged $2 \mathrm{~cm}$ below the water surface, with the water temperature maintained at $21 \pm 1^{\circ} \mathrm{C}$. Four points around the edge of the pool were designated as North (N), South (S), East (E) and West (W). A video camera was mounted above the center of the pool and all performance was recorded and then analyzed by a computerized video imaging analysis system (Institute of Material Medical, Chinese Academy of Medical Sciences, Beijing, China). At the beginning of the trials, the rats were free to swim for 2 min and the swimming distances were recorded. At first, the rats were provided an escape latency trial once per day for four days. The rats were placed from E, S, W and N one by one every day. If the rat did not find the escape platform within the allotted time ( $2 \mathrm{~min}$ ), the record was designated as $120 \mathrm{sec}$. On day 15 , the platforms were removed and the rats were placed into the maze as the escape latency trial. A $120 \mathrm{sec}$ probe trial was performed and the number of crossings over the target quadrant was recorded. Each test was repeated four times for each rat at every time-point. The time scale of the MWM test is illustrated in Fig. 1.

Synapse density observed by transmission electron microscopy. The synapse density in hippocampal CA1 and cortex parietal was observed with a transmission electron microscope (H7650, Hitachi, Ltd., Tokyo, Japan). Briefly, following regular fixation, embedding and ultra-thin section, the brain sections of each rat were placed onto three copper grids. Each grid was scanned to capture 4-5 images, with a total of 10-15 pictures for each rat brain. The numerical density of synapses per unit volume, $\mathrm{N}_{\mathrm{v}}$, was calculated using the following formula: $\mathrm{N}_{\mathrm{v}}=\mathrm{Q}^{-} / \mathrm{V}_{\mathrm{dis}}$, where $\mathrm{Q}^{-}$is the mean number of synapses counted in each sector and $V_{\text {dis }}$ is the mean sector volume. The total number of synapses, $\mathrm{N}_{\mathrm{syn}}$, was calculated for each case using the following formula: $\mathrm{N}_{\mathrm{syn}}=\mathrm{N}_{\mathrm{v}} \cdot \mathrm{V}_{\text {ref }}$ 
Table I. Polymerase chain reaction primers, fragment length and annealing time of GPx-1, CAT and $\beta$-actin.

\begin{tabular}{|c|c|c|c|}
\hline Gene & Primer sequence & Product (bp) & Annealing temperature $\left({ }^{\circ} \mathrm{C}\right)$ \\
\hline CAT & $\begin{array}{l}\text { F: 5'-CTATCCTGACACTCACCGCCAT-3' } \\
\text { R: 5'-TTCTTGACCGCTTTCTTCTGGA-3' }\end{array}$ & 372 & 58 \\
\hline GPX-1 & $\begin{array}{l}\text { F: 5'-CTC GGTTTCCCGTGCAATCAG-3' } \\
\text { R: 5'-GTGCAGCCAGTAATCACCAAG-3' }\end{array}$ & 431 & 65 \\
\hline$\beta$-actin & $\begin{array}{l}\text { F: 5'-CTGACCGAGCGTGGCTAC-3' } \\
\text { R: 5'-CCTGCTTGCTGATCCACA-3' }\end{array}$ & 505 & 58 \\
\hline
\end{tabular}

GPx-1, glutathione peroxidase-1; CAT, catalase.

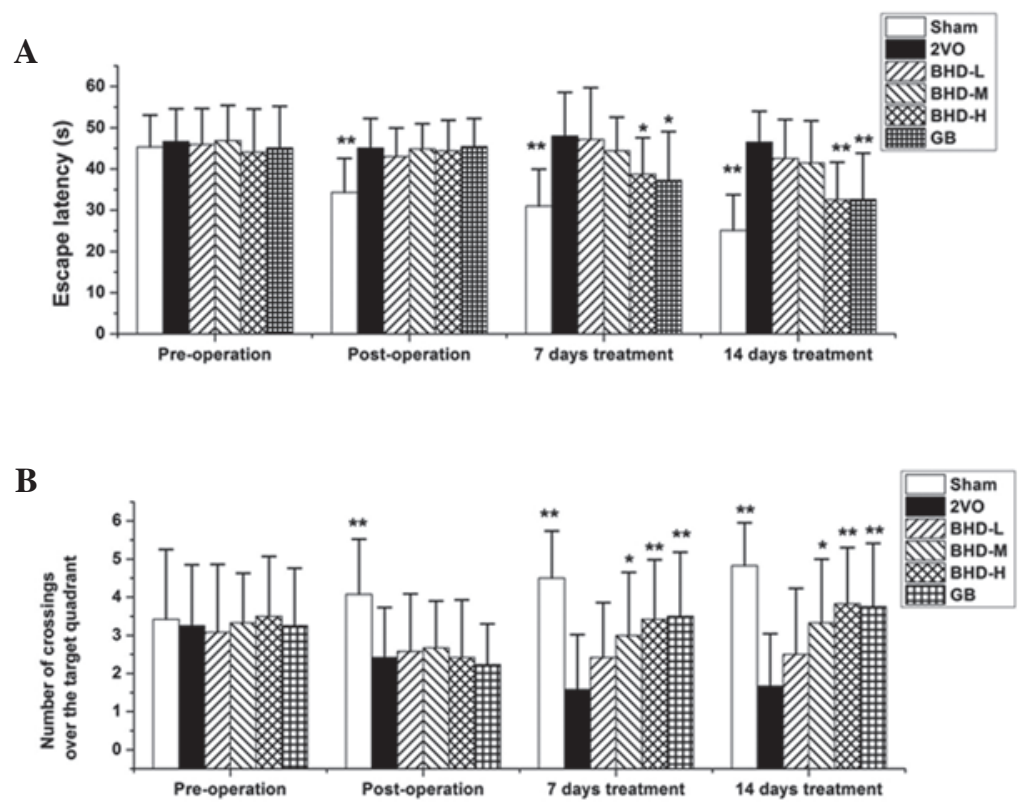

Figure 2. BHD increased spatial learning and memory in 2VO model rats. (A) Morris water maze was performed prior to the surgery, post-surgery without treatment, and on days 7 and 14 following administration, respectively. Escape latency time was calculated and illustrated in the bar chart. (B) The number of crossing over the target quadrant in probe trials was measured in each group. $\mathrm{n}=12$ per group, ${ }^{*} \mathrm{P}<0.05$ and ${ }^{* *} \mathrm{P}<0.01 \mathrm{vs}$. the $2 \mathrm{VO}$ group. BHD-L/M/H, Bushen Huoxue decoction-low/moderate/high dose; GB, Ginkgo biloba, 2VO, occlusion of bilateral common carotid arteries.

ELISA. The hippocampus homogenates were isolated and stored at $-80^{\circ} \mathrm{C}$ until use. Superoxide dismutase (SOD) and catalase (CAT) levels were determined with respected ELISA kits (Hufeng Tech, Shanghai, China) according to manufacturer's instructions.

Western blot analysis. The denatured protein samples were resolved by SDS-PAGE and transferred to polyvinylidene fluoride membranes (Millipore, Billerica, MA, USA). Following blocking, the membranes were incubated overnight at $4^{\circ} \mathrm{C}$ with primary antibodies $[\beta$-actin and CAT monoclonal rabbit anti-mouse antibodies, 1:1,000 dilution, Cell Signaling Technology, Inc., Danvers, MA, USA; polyclonal rabbit glutathione peroxidase-1 (GPx-1) antibody, 1:1,000 dilution, Abcam, Cambridge, UK] followed by incubation with the goat anti-rabbit horseradish peroxidase-conjugated secondary antibodies (1:2,000 dilution; Santa Cruz Biotechnology Inc., Santa Cruz, CA, USA). Chemiluminescence detection was performed using enhanced chemiluminescence advance western blotting detection reagents (BD Biosciences, San Jose, CA, USA). Band intensities were measured with the SX-300 image analysis system (Bio-Rad Laboratories, Hercules, CA, USA).

Quantitative polymerase chain reaction ( $q P C R)$. Total RNA was extracted from the hippocampi with TRIzol reagent (Invitrogen Life Technologies, Carlsbad, CA, USA) according to the manufacturer's instructions. Reverse transcription was performed using the AMV reverse transcription system (Fermentas, Burlington, Canada). Amplification of cDNA fragments of CAT, GPx-1 and $\beta$-actin was performed using the Green PCR Master Mix kit (Fermentas). DNA was amplified immediately with a single cycle at $94^{\circ} \mathrm{C}$ for $5 \mathrm{~min}$, 30 cycles at $94^{\circ} \mathrm{C}$ for $30 \mathrm{sec}$, annealing temperature of each gene for $30 \mathrm{sec}$ and $72^{\circ} \mathrm{C}$ for $30 \mathrm{sec}$ followed by a final extension step $72^{\circ} \mathrm{C}$ for $10 \mathrm{~min}$. Ethidium bromide-stained gels were scanned and qualified using Tanon Image software (Science \& Technology Co. Ltd., Shanghai, China). The 
A
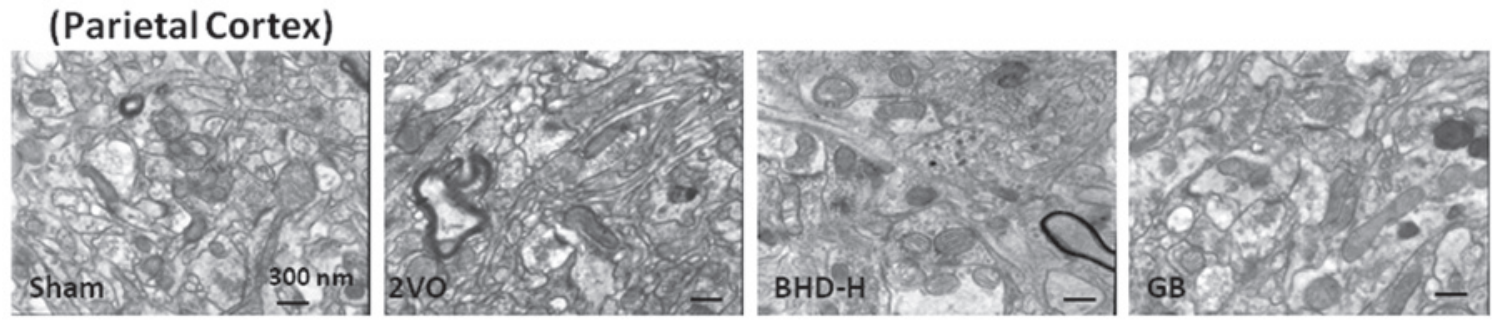

B

(Hippocampus CA1)
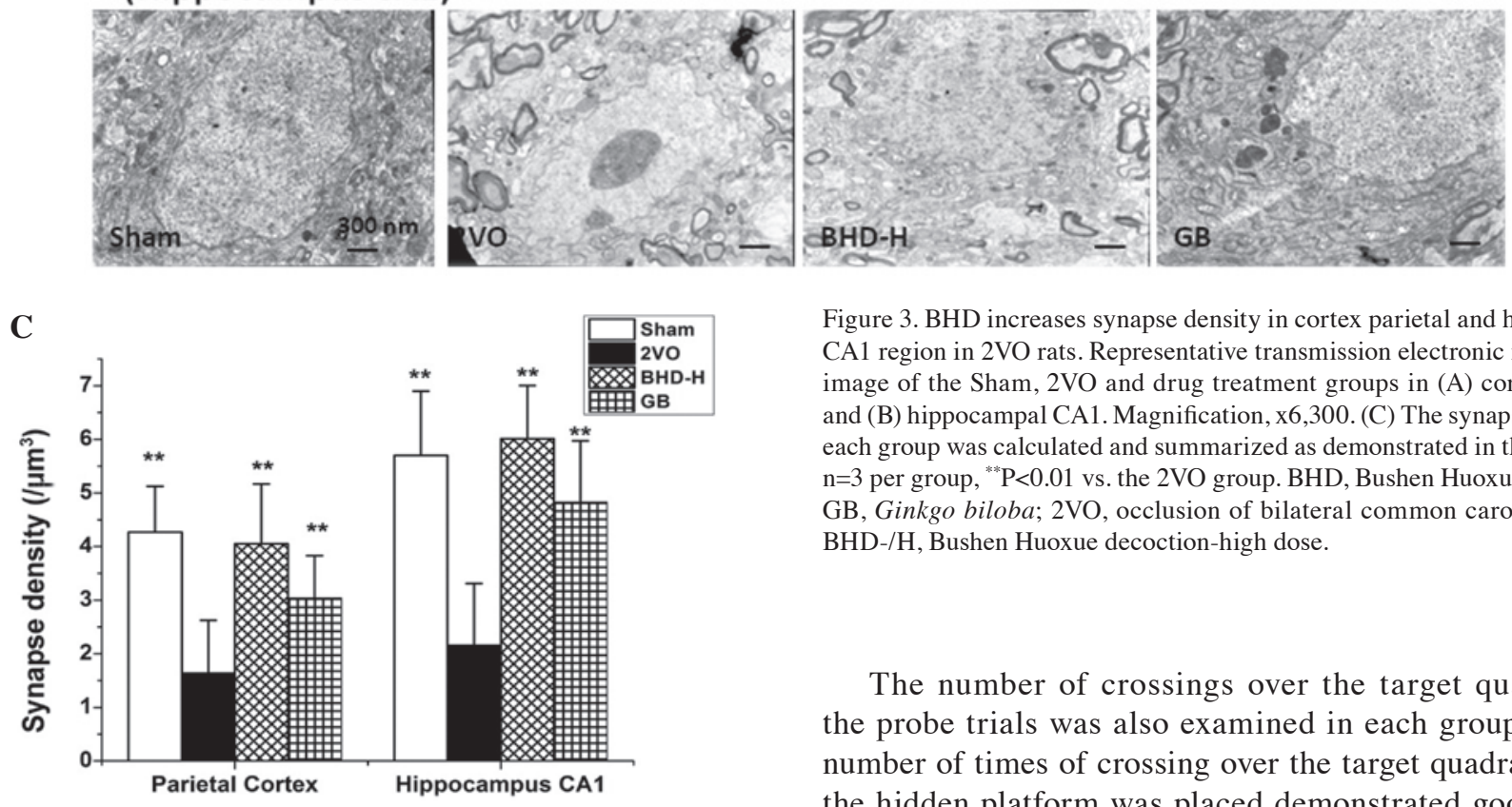

Figure 3. BHD increases synapse density in cortex parietal and hippocampal $\mathrm{CA} 1$ region in $2 \mathrm{VO}$ rats. Representative transmission electronic microscopic image of the Sham, 2VO and drug treatment groups in (A) cortex parietal and (B) hippocampal CA1. Magnification, x6,300. (C) The synapse density in each group was calculated and summarized as demonstrated in the bar chart. $\mathrm{n}=3$ per group, ${ }^{* *} \mathrm{P}<0.01$ vs. the $2 \mathrm{VO}$ group. $\mathrm{BHD}$, Bushen Huoxue decoction; $\mathrm{GB}$, Ginkgo biloba; $2 \mathrm{VO}$, occlusion of bilateral common carotid arteries; BHD-/H, Bushen Huoxue decoction-high dose.

The number of crossings over the target quadrant in the probe trials was also examined in each group. A large number of times of crossing over the target quadrant where the hidden platform was placed demonstrated good spatial learning and memory. Fig. 2B demonstrated that the rats in the $2 \mathrm{VO}$ group required less time than the sham group intensity of each band was normalized against the intensity of $\beta$-actin. The PCR primers, fragment length and annealing time of GPX-1, CAT and $\beta$-actin are summarized in Table I.

Statistical analysis. Statistical analysis was performed with SPSS 12.0 (SPSS, Inc, Chicago, IL, USA). Data are expressed as the mean \pm standard deviation. Analysis of variance was used to determine statistical significance. $\mathrm{P}<0.05$ was considered to indicate a statistically significant difference.

\section{Results}

BHD improves spatial learning and memory of rats with $2 V O$ in $M W M$. To observe whether BHD improves cognitive deficits, the classical MWM test was used to examine spatial learning and memory, as described in materials and methods above. As demonstrated in Fig. 2A, the escape latency to find the hidden platform in the sham group decreased gradually, while the $2 \mathrm{VO}$ rats spent more time in the training trials ( $\mathrm{P}<0.01$, compared with the model group). These observations are consistent with the results of behavioral tests reported elsewhere (16). The rats in the BHD-M, BHD-H dosage of BHD and positive control (GB) groups demonstrated improved cognitive abilities compared with the $2 \mathrm{VO}$ rats from days 7 and 14 of the tests $(\mathrm{P}<0.05$ or $\mathrm{P}<0.01$, compared with the model group), whereas low-dose BHD did not induce any significant improvements. $(\mathrm{P}<0.01)$, and the number of crossings over the target quadrant increased following treatment with BHD as well as GB. The results suggested that rats with $2 \mathrm{VO}$ treated with BHD-M or BHD-H exhibited a significant increase in the number crossings over the target quadrant $(\mathrm{P}<0.05$, compared with the model group), particularly at a high dose $(\mathrm{P}<0.01$, compared with the model group). Overall, these results demonstrated that BHD dose-dependently improved cognitive deficits.

$B H D$ increases the number of synapses in hippocampal CAI regions and the parietal cortex in rats with $2 \mathrm{VO}$. Cognitive impairment is strongly associated with a reduction in the number of synapses. (17) To detect whether the resultant loss in the number of synapses due to cerebral hypoperfusion could be alleviated by BHD administration, the synapses in the hippocampal CA1 region and parietal cortex in rats with $2 \mathrm{VO}$ were observed using transmission electron microscopy, counting the number of synapses in each specified area. The results in Fig. 3 revealed that the synapse density in both areas was reduced in the $2 \mathrm{VO}$ model, which is consistent with a previous study (18). Notably, both the high dosage of BHD and GB treatment reversed the synaptic impairments observed in the parietal cortex and hippocampal CA1 regions.

Expression of antioxidants increases following BHD treatment in the hippocampi of rats with $2 \mathrm{VO}$. To further study the molecular mechanism underlying the BHD-induced cognitive 
Table II. Effects of BHD on the content of SOD, CAT, GSH and MDA in the 2VO rats.

\begin{tabular}{lcccc}
\hline Groups & MDA $(\mathrm{nmol} / \mathrm{mg})$ & SOD $(\mathrm{U} / \mathrm{mg})$ & CAT $(\mathrm{U} / \mathrm{mg})$ & $\mathrm{GSH}(\mu \mathrm{g} / \mathrm{mg})$ \\
\hline Sham & $8.32 \pm 0.52^{\mathrm{b}}$ & $115.03 \pm 9.67^{\mathrm{b}}$ & $6.94 \pm 0.69^{\mathrm{b}}$ & $104.90 \pm 13.33^{\mathrm{b}}$ \\
2 VO & $11.49 \pm 0.54$ & $100.19 \pm 8.53$ & $5.20 \pm 0.35$ & $79.54 \pm 9.85$ \\
BHD-L & $10.79 \pm 1.45$ & $109.16 \pm 2.83$ & $6.01 \pm 0.63^{\mathrm{a}}$ & $88.97 \pm 7.99^{\mathrm{a}}$ \\
BHD-M & $9.81 \pm 0.67^{\mathrm{b}}$ & $110.68 \pm 12.43^{\mathrm{a}}$ & $6.51 \pm 0.79^{\mathrm{b}}$ & $94.41 \pm 16.66^{\mathrm{a}}$ \\
BHD-H & $8.49 \pm 0.49^{\mathrm{b}}$ & $130.60 \pm 9.13^{\mathrm{b}}$ & $6.65 \pm 0.43^{\mathrm{b}}$ & $98.47 \pm 10.16^{\mathrm{b}}$ \\
GB & $8.90 \pm 0.53^{\mathrm{b}}$ & $110.84 \pm 7.11^{\mathrm{a}}$ & $6.05 \pm 0.63^{\mathrm{a}}$ & $92.63 \pm 7.36^{\mathrm{a}}$ \\
\hline
\end{tabular}

Compared with model group ${ }^{\mathrm{a}} \mathrm{P}<0.05,{ }^{\mathrm{b}} \mathrm{P}<0.01$ and $\mathrm{n}=12$. BHD, Bushen Huoxue decoction; GB, Ginkgo biloba; SOD, superoxide dismutase; CAT, catalase; GSH, glutathione; 2VO, occlusion of bilateral common carotid arteries; BHD-L/M/H, Bushen Huoxue decoction-low/moderate/high dose.

A

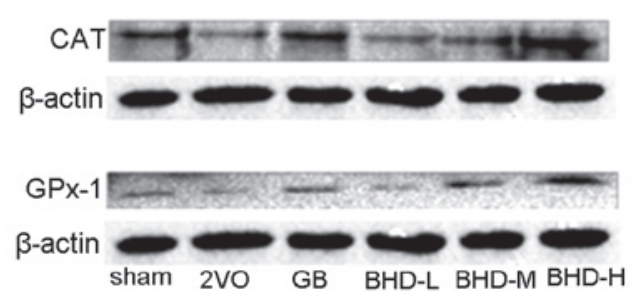

C
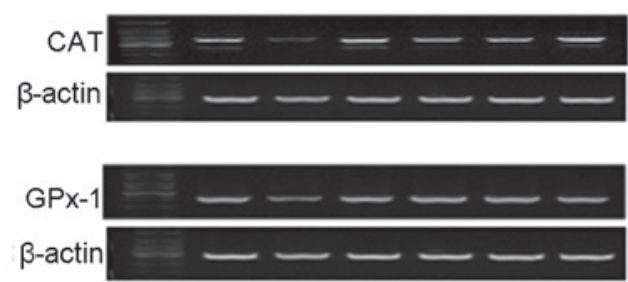

B

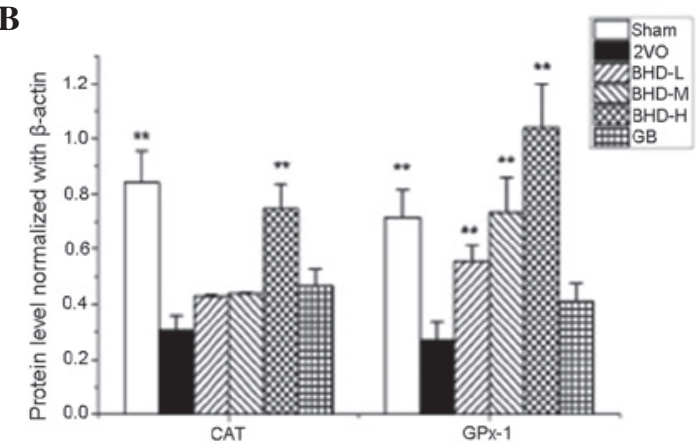

D

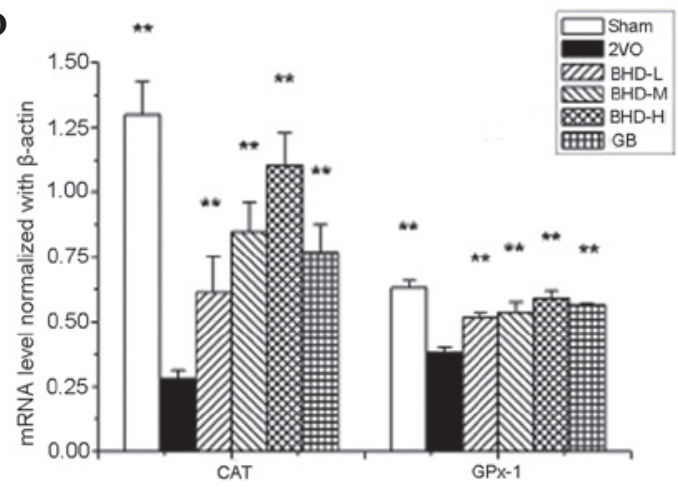

Figure 4. BHD increases the mRNA and protein expression levels of GPx-1 and CAT in the hippocampus. Protein levels of GPx-1 and CAT in the hippocampus were (A) assessed using western blot analysis and (B) quantified. mRNA levels of GPx-1 and CAT in hippocampus were (C) observed using quantitative polymerase chain reaction and (D) quantified. $\mathrm{n}=3$ per group, ${ }^{*} \mathrm{P}<0.05$ and ${ }^{* *} \mathrm{P}<0.01$ vs. the 2 VO group. GB, Ginkgo biloba; GPx-1, glutathione peroxidase-1; $\mathrm{CAT}$, catalase; 2VO, occlusion of bilateral common carotid arteries; BHD-L/M/H, Bushen Huoxue decoction-low/moderate/high dose.

improvement, antioxidant levels and activities were examined. Increased levels of malondialdehyde (MDA) and a decrease in the activity of SOD, CAT and glutathione (GSH) in cerebral hypoperfusion induced by $2 \mathrm{VO}$ was observed $(\mathrm{P}<0.01$, compared with the sham group; Table II). Both the GB and BHD groups demonstrated a significant reduction in the MDA levels in the hippocampus, with the GB, BHD-M and BHD-H groups exhibiting significantly decreased MDA levels $(\mathrm{P}<0.01$, compared with the model group). In addition, compared with the model group, both GB and BHD increased the activities of SOD, CAT and GSH $(\mathrm{P}<0.01)$. 2VO markedly downregulated the mRNA and protein expression levels of CAT and GPx-1 in the hippocampus $(\mathrm{P}<0.01$, compared with the sham group; Fig. 4). Both GPX-1 and CAT expression increased at the mRNA and protein levels in a dose-dependent manner following BHD treatment, which is consistent with the ELISA data.

\section{Discussion}

Cognitive impairment of all types, from mild to severe associated with cerebrovascular damage, is defined as vascular cognitive impairment, as first proposed by O'Brien et al (19) which is characterized as the prodromal stages of ischemic $\mathrm{VaD}$ (20). Accumulating evidence has implicated VCI as a prominent cause of cognitive decline in the elderly $(21,22)$.

The MWM test is a classic method to examine spatial learning and memory function (23). In the present study, to exclude the possibility that the $2 \mathrm{VO}$ model and drug administra- 
tion affect motoric disability rather than cognitive decline, the swimming velocity was measured prior to the formal MWM test. There was no significant difference in the time to swim the distance $(120 \mathrm{sec})$ between the groups $(\mathrm{P}>0.05$, data not shown). This quality control ensured the equal basic physical and motoric ability in the water for each group. Next, escape latency and probe trials were conducted to determine the acquisition and retention ability of the experimental rats. The results demonstrated that in rats with cerebral hypoperfusion, treatment with BHD significantly increased the number of crossings over the target quadrant and decreased the escape latency to reach the platform, particularly at the mid and high dosages, indicating that BHD improved cognitive functioning in the rats with $2 \mathrm{VO}$.

Synapse formation and plasticity are necessary for memory recall and consolidation. Previous studies have revealed that synapse loss is associated with cognitive impairment observed in various cognitive dysfunction models, including AD $(24,25)$, middle cerebral artery occlusion (26) and 2VO (27). In the present study, BHD treatment evidently alleviated the synaptic loss induced by cerebral hypoperfusion. Reversing this pathological change may have largely contributed to the memory recall and improvement of cognitive impairment.

Increased levels of oxidative stress and antioxidant deficiencies may pose as risk factors for cognitive decline (28). Chronic vascular hypoperfusion induces oxidative stress and brain energy failure and leads to neuronal death. This is because the brain is particularly susceptible to free radical attack, which generates more toxins per gram of tissue than any other organ in the body (29). Therefore, anti-oxidative therapy is considered as a good way to improve cognitive deficits (30). MDA is a marker of lipid peroxidation and the levels of MDA indicate the degree of oxidative stress (31). In the antioxidant enzyme defense system, SOD catalyzes the formation of hydrogen peroxide from superoxide radicals, while CAT and GPx prevent or remove toxic hydroxyl radicals generated by hydrogen peroxide (32).

The most significant ingredient of BHD, Epimedium, an important Chinese Herb which nourishes the kidney and enhances brain functioning, has been proved to possess antioxidant activities $(33,34)$. The ministerial drug Salvia Miltrorrhiza, which has an activating effect on the blood and is often used for cerebrovascular diseases, was also demonstrated to have anti-oxidant activities (35). The extract of GB leaves, the positive control used in this study, was found to possess cognitive enhancing effects associated with its antioxidant properties (36). In the present study, BHD reduced MDA levels and enhanced the activity of SOD, CAT and GSH in the hippocampi of rats with 2VO. BHD treatment also increased the expression of SOD and GPx at both the protein and mRNA levels. These results indicated that the role of BHD improving learning and memory is associated with the contents or activity of antioxidants and antioxidative enzymes.

In conclusion, the present study evidently demonstrated that BHD improved learning and memory deficits induced by cerebral hypoperfusion, and the restoration of elevated antioxidants and synapse density may have contributed to this improvement. The present study provided evidence that will facilitate developing BHD as a preventive or therapeutic for $\mathrm{VaD}$.

\section{Acknowledgements}

This study was supported by the Developmental Fund of Chen Keji Integrative Medicine, no. CKJ2010025, and the Key Foundation of Society Development in Fujian province, no. 2013 Y0059.

\section{References}

1. Gorelick PB and Pantoni L: Advances in vascular cognitive impairment. Stroke 44: 307-308, 2013.

2. Chui HC: Vascular cognitive impairment: today and tomorrow. Alzheimers Dement 2: 185-194, 2006.

3. Moorhouse P and Rockwood K: Vascular cognitive impairment: current concepts and clinical developments. Lancet Neurol 7: 246-255, 2008.

4. Consoli A, Pasi M and Pantoni L: Vascular mild cognitive impairment: concept, definition, and directions for future studies. Aging Clin Exp Res 24: 113-116, 2012.

5. Dichgans M and Zietemann V: Prevention of vascular cognitive impairment. Stroke 43: 3137-3146, 2012.

6. Kalaria RN: Cerebrovascular disease and mechanisms of cognitive impairment: evidence from clinicopathological studies in humans. Stroke 43: 2526-2534, 2012.

7. Luckhaus C, Flüb MO, Wittsack HJ, et al: Detection of changed regional cerebral blood flow in mild cognitive impairment and early Alzheimer's dementia by perfusion-weighted magnetic resonance imaging. Neuroimage 40: 495-503, 2008.

8. Panza F, Frisardi V, Capurso C, et al: Metabolic syndrome and cognitive impairment: current epidemiology and possible underlying mechanisms. J Alzheimers Dis 21: 691-724, 2010.

9. Clare R, King VG, Wirenfeldt M and Vinters HV: Synapse loss in dementias. J Neurosci Res 88: 2083-2090, 2010.

10. Jesky $R$ and Hailong $C$ : Are herbal compounds the next frontier for alleviating learning and memory impairments? An integrative look at memory, dementia and the promising therapeutics of traditional chinese medicines. Phytother Res 25: 1105-1118, 2011.

11. Liu X, Du J, Cai J, Xu G, Lin A and Teng Q: Clinical systematic observation of Kangxin capsule curing vascular dementia of senile kidney deficiency and blood stagnation type. J Ethnopharmacol 112: 350-355, 2007.

12. Moss G: The adequacy of the cerebral collateral circulation: tolerance of awake experimental animals to acute bilateral common carotid artery occlusion. J Surg Res 16: 337-338, 1974.

13. Brondino N, De Silvestri A, Re S, et al: A Systematic review and meta-analysis of ginkgo biloba in neuropsychiatric disorders: from ancient tradition to modern-day medicine. Evid Based Complement Alternat Med 2013: 915691, 2013.

14. Ihl R: Effects of Ginkgo biloba extract EGb 761 in dementia with neuropsychiatric features: review of recently completed randomised, controlled trials. Int J Psychiatry Clin Pract 1 (Suppl 1): 8-14, 2013.

15. Morris R: Developments of a water-maze procedure for studying spatial learning in the rat. J Neurosci Methods 11: 47-60, 1984.

16. Feng Z, Lu Y, Wu X, et al: Ligustilide alleviates brain damage and improves cognitive function in rats of chronic cerebral hypoperfusion. J Ethnopharmacol 144: 313-321, 2012.

17. Scheff SW, Price DA, Schmitt FA, et al: Synaptic alterations in CA1 in mild Alzheimer disease and mild cognitive impairment. Neurology 68: 1501-1508, 2007.

18. Wang X, Xing A, Xu C, Cai Q, Liu H and Li L: Cerebrovascular hypoperfusion induces spatial memory impairment, synaptic changes, and amyloid- $\beta$ oligomerization in rats. J Alzheimers Dis 21: 813-822, 2010.

19. O'Brien JT, Erkinjuntti T, Reisberg B, et al: Vascular cognitive impairment. Lancet Neurol 2: 89-98, 2003.

20. Ramos-Estébanez C, Moral-Arce I, Muñoz-Arrondo R, et al: Vascular cognitive impairment: prodromal stages of ischemic vascular dementia. Dement Geriatr Cogn Disord 25: 451-460, 2008 . 
21. Hachinski V, Iadecola C, Petersen RC, et al: National Institute of Neurological Disorders and Stroke-Canadian Stroke Network vascular cognitive impairment harmonization standards. Stroke 37: 2220-2241, 2006.

22. Bowler JV: Modern concept of vascular cognitive impairment. Br Med Bull 83: 291-305, 2007.

23. D'Hooge R and De Deyn PP: Applications of the Morris water maze in the study of learning and memory. Brain Res Brain Res Rev 36: 60-90, 2001

24. Dong H, Martin MV, Chambers S and Csernansky JG: Spatial relationship between synapse loss and beta-amyloid deposition in Tg2576 mice. J Comp Neurol 500: 311-321, 2007.

25. Marcello E, Epis R, Saraceno C and Di Luca M: Synaptic dysfunction in Alzheimer's disease. Adv Exp Med Biol 970 573-601, 2012.

26. Yao ZB, Li X and Xu ZC: GABAergic and asymmetrical synapses on somata of GABAergic neurons in CA1 and CA3 regions of rat hippocampus. A quantitative electron microscopic analysis. Stroke 27: 1411-1415, 1996.

27. Hai J, Yu F, Lin Q and Su SH: The changes of signal transduction pathways in hippocampal regions and postsynaptic densities after chronic cerebral hypoperfusion in rats. Brain Res 1429: 9-17, 2012.

28. Berr C, Balansard B, Arnaud J, Roussel AM and Alperovitch A Cognitive decline is associated with systemic oxidative stress: the EVA study. Etude du Vieillissement Artériel. J Am Geriatr Soc 48: 1285-1291, 2000 .

29. Reiter RJ: Oxidative processes and antioxidative defense mechanisms in the aging brain. FASEB J 9: 526-533, 1995.
30. Mecocci P, Mariani E, Cornacchiola V and Polidori MC: Antioxidants for the treatment of mild cognitive impairment. Neurol Res 26: 598-602, 2004.

31. Bennett S, Grant MM and Aldred S: Oxidative stress in vascular dementia and Alzheimer's disease: a common pathology. J Alzheimers Dis 17: 245-257, 2009.

32. López N, Tormo C, De Blas I, Llinares I and Alom J: Oxidative stress in Alzheimer's disease and mild cognitive impairment with high sensitivity and specificity. J Alzheimers Dis 33: 823-829, 2013.

33. Yuan D, Wang $\mathrm{H}, \mathrm{He} \mathrm{H}$, et al: Protective effects of total flavonoids from Epimedium on the male mouse reproductive system against cyclophosphamide-induced oxidative injury by up-regulating the expressions of SOD3 and GPX1. Phytother Res 28: 88-97, 2014

34. Cheng H, Feng S, Jia X, Li Q, Zhou Y and Ding C: Structural characterization and antioxidant activities of polysaccharides extracted from Epimedium acuminatum. Carbohydr Polym 92: 63-68, 2013.

35. Wu YC and Hsieh CL: Pharmacological effects of Radix Angelica Sinensis (Danggui) on cerebral infarction. Chin Med 6: 32, 2011.

36. Mansour SM, Bahgat AK, El-Khatib AS and Khayyal MT: Ginkgo biloba extract (EGb 761) normalizes hypertension in $2 \mathrm{~K}, 1 \mathrm{C}$ hypertensive rats: role of antioxidant mechanisms, ACE inhibiting activity and improvement of endothelial dysfunction. Phytomedicine 18: 641-647, 2011. 